\title{
Análisis factorial exploratorio de una prueba de personalidad en la Unidad Militar de Emergencias
}

\author{
Samper Lucena E. ${ }^{1}$, Robles Sánchez JI. ${ }^{2}$ \\ Sanid. mil. 2014; 70 (4): 256-262; ISSN: 1887-8571
}

\begin{abstract}
RESUMEN
En el artículo se presenta un estudio factorial exploratorio de una prueba de personalidad que se utiliza en la Unidad Militar de Emergencias. Se realiza sobre una muestra de 1661 militares que trabajan en situaciones de emergencias y catástrofes. El estudio se ha realizado en cuatro fases características: El cálculo de una matriz capaz de expresar la variabilidad conjunta de todas las variables, la extracción del número óptimo de factores, la rotación de la solución para facilitar su interpretación y la estimación de las nuevas puntuaciones. Los resultados obtenidos muestran dos factores de personalidad principales, el primer factor se le ha denominado “emocional", aglutina las variables de depresión, neuroticismo y psicoticismo de la prueba, el segundo factor se le ha denominado "social" y contiene las variables de psicopatía y sociabilidad, en ambos casos los índices de varianza explicada son mejorables y el índice de falibilidad de la prueba fue muy bajo. La prueba ha mostrado validez ecológica en su utilización en la unidad, sin embargo es necesario mas estudios para conseguir evaluar los rasgos personales de forma más precisa, útil y actual, consiguiendo realizar mejores predicciones conductuales y en última instancia mejorar la operatividad de las Unidades.
\end{abstract}

PALABRAS CLAVE: evaluación psicológica, personalidad, análisis factorial.

\section{Exploratory factor analysis of a personality test in emergency military unit}

SUMMARY: the article presents a study factorial exploratory of a test of personality that is in use in the Military Unit of Emergencies. It is realized on a sample of 1661 Military men. The study has been realized in four typical phases: The calculation of a counterfoil capable of expressing the joint variability of all the variables, the extraction of the ideal number of factors, the rotation of the solution to facilitate his interpretation and the estimation of the new punctuations. The obtained results show two principal factors of personality, the first factor has named him "emotionally", agglutinates the variables of depression, neuroticism and psychoticism of the test, the second factor has named him "socially" and contains the variables of psychopaty and sociableness of the test, in both cases the indexes of explained variance are improvable and the index of fallibility of the test was very low. The test has showed ecological validity in his utilization in the Unit nevertheless it is a necessary mas studies to manage to evaluate the personal features of the most precise, useful and current form, managing to realize better behavioral predictions and in last instance to improve the operability of the Units.

KEYWORDS: psychological assessment, personality, factor analysis.

\section{INTRODUCCIÓN}

Se ha utilizado de una forma común el término de personalidad. Desde una perspectiva histórica la palabra personalidad deriva de una persona, término griego que representa una máscara que utilizaban los actores en sus representaciones en el teatro, esto sugería que la persona era "en apariencia" y en realidad, debajo de la máscara había otra serie de rasgos que identificaban realmente a la persona.

A comienzos de la Edad Media y por asimilación del término prosopón (máscara) a hypóstasis (sustrato o base) el término persona perdió su connotación de ilusión y empezó a representar a la persona real y sus características explicitas y manifiestas. Si

\footnotetext{
${ }^{1}$ Cte. Psi. Unidad Militar de Emergencias.

${ }^{2}$ Cte. Psi. Hospital Central de la Defensa Gómez Ulla. Servicio Psicología. Madrid. España.
}

Dirección para correspondencia: Eduardo Samper Lucena. Cte. Psi. Unidad Militar de Emergencias UME. CG. Teléfono 917487119. Email esamluc@ea.mde.es.

Recibido: 16 de septiembre de 2013

Aceptado: 21 de enero de 2014 se plantea el tema de responder a la cuestión ¿qué es la personalidad?, aparecen dificultades en encontrar una respuesta única, su significado no es simple, ni uniforme, ni siquiera a nivel etimológico ${ }^{1}$.

Es un concepto básico en psicología que ha recibido numerosas definiciones. La personalidad se puede definir como el conjunto de características o patrón de sentimientos, pensamientos, actitudes o hábitos, ligados al comportamiento, que persiste en el tiempo y las situaciones. Esto Se refiere a un patrón complejo de características psicológicas profundamente arraigadas, que son en su mayor parte inconscientes y difíciles de cambiar, y se expresan automáticamente en casi todas las áreas de funcionamiento del individuo. Estos rasgos internos surgen de determinantes biológicos o temperamento y de aprendizajes o carácter que componen el patrón idiosincrásico de sentir, pensar, afrontar y comportarse de un sujeto ${ }^{2}$.

Esto hace que unos individuos sean diferentes a otros. Siguiendo al DSM-IV TR $(2002)^{3}$ los rasgos de personalidad, se definen como patrones persistentes de formas de percibir, relacionarse y pensar sobre el entorno y sobre uno mismo que se ponen de manifiesto en una amplia gama de contextos sociales 
y personales. Los rasgos de personalidad constituyen trastornos de personalidad cuando son inflexibles, no se adaptan a las normas sociales y cuando causan un deterioro funcional significativo o un malestar subjetivo.

La psicología de la personalidad, es la disciplina que se ocupa del estudio psicológico de la persona ${ }^{1}$. Una de sus características es su heterogeneidad teórica y de enfoques. Eysenck ${ }^{4-6}$ por ejemplo, propone un modelo jerárquico de personalidad consistente en conductas y conjuntos de conductas que varían en el nivel de generalidad e importancia, el nivel más bajo está representado por el nivel de respuestas especificas, que se refieren a actos o cogniciones, como por ejemplo la respuesta a un test o a experiencias de la vida diaria que pueden observarse una vez y que pueden o no, ser características del sujeto.

En el segundo nivel, se encuentran las denominadas respuestas habituales, que son respuestas específicas que tienden a ocurrir de forma reiterada bajo circunstancias similares. Este nivel del hábito representa el nivel de organización más bajo y puede medirse en términos de coeficiente de fiabilidad.

En el tercer nivel, los actos habituales se organizan en rasgos. Los rasgos (sociabilidad, irritabilidad, impulsividad...), son constructos teóricos que se basan en las intercorrelaciones significativas que se observan entre un número de respuestas habituales. Este nivel correspondería con lo que se denominan factores de primer orden.

El último nivel, se caracteriza por la organización de los rasgos en un tipo general. Presenta el nivel más amplio de organización y también se establece a partir de correlaciones significativas, pero en este caso, las correlaciones observadas entre los diferentes rasgos dan lugar a un constructo de orden superior. Los tipos o dimensiones en el modelo de la personalidad de Eysenck, son tres: extraversión - introversión, neuroticismo y psicoticismo ${ }^{7}$.

Siguiendo otro modelo "el biopsicosocial", se puede hablar de tres componentes en los sujetos:

a) Un componente biológico básico, que se ha demostrado asociado, a través de los estudios clásicos de Eysenck ${ }^{8}$, de Zuckerman ${ }^{9}$ o en nuestro país de Labrador ${ }^{10,11}$, con los factores de personalidad. Sujetos con un alto índice de psicopatía por ejemplo, poseen un sistema activador de la conducta particular. Esta forma de comportamiento se asocia en el sistema neuropsicológico que implica principalmente a la amígdala y es sensible a estímulos incondicionados. En una revisión de $\operatorname{Corr}^{12}$ se asocia el psicoticismo principalmente con el sistema de lucha y huida, mientras que antes se asociaba a los sistemas de activación e inhibición de la conducta ${ }^{13}$. Sin embargo cierto nivel de estas características personales también se encuentra entre población "normal", por ejemplo entre personas jóvenes adolescentes ${ }^{14}$ que se puede relacionar con la población analizada en este estudio. Habrá que tener en cuenta tal como señalan Herrero y Colom que la influencia de factores de personalidad no implica imposibilidad de cambio ${ }^{15}$.

b) Un componente psicológico: donde numerosos estudios sobre los factores de estrés también inciden en señalar a los factores personales como recursos para hacer frente a situaciones complicadas ${ }^{10}$ y siguiendo la propuesta de Roberston ${ }^{16}$ el papel que juegan los estresores laborales en la salud, la satisfacción laboral y la intención de abandono del puesto de trabajo en el personal militar, se encuentran mediados por variables de tipo personal.

c) Un componente social: medido por la extraversión o sociabilidad y la psicopatía. Son ya clásicos los trabajos en los que se ha asociado la psicopatía con comportamientos sociales ${ }^{17-19}$. Hay autores que señalan un componente de psicopatía en sujetos que son buscadores de sensaciones, normalmente esto se ha asociado a sujetos problemáticos socialmente más que a población general ${ }^{20-22}$. Siendo también contemplados en los criterios diagnósticos del "Trastorno Antisocial de la Personalidad" de la Asociación Americana de Psicología (APA) ${ }^{3}$ y de la psicopatía ${ }^{23}$. Sujetos con un alto componente psicopatológico se podrían considerar más vulnerables en este sentido siguiendo la teoría de Lykken $^{24}$ y Gray ${ }^{25}$.

\section{¿Por qué evaluar la personalidad?}

En las fuerzas armadas (FAS) la evaluación de los factores de personalidad se lleva haciendo a lo largo de toda su historia, en la actualidad es un cometido de la psicología integrada en el cuerpo militar de sanidad. Se asocia la evaluación de los factores de personalidad con los procesos de selección del personal donde se tiene en cuenta tanto factores intelectuales como personales, con el objetivo de adaptar la persona al puesto de trabajo. Otra utilidad de estos factores está asociada a la salud, su atención y prevención, o en temas relacionados con la seguridad y la operatividad de las unidades. El mismo Manual de Procedimientos de Prevención de Riesgos Laborales, elaborado por el Instituto Nacional de Seguridad e Higiene en el Trabajo ${ }^{26}$ recoge explícitamente que se deberá cuidar la adecuación de la persona al perfil del puesto, incluyendo los criterios de seguridad y salud.

Una vez que el personal se integra en las unidades de las FAS, el objetivo es que estas unidades posean altos grados de operatividad y que desarrollen su misión en las mejores condiciones. A veces esto conlleva realizar el trabajo en condiciones complicadas de combate, de emergencias o catástrofes etc. Esto produce un gran desgaste que es un índice de bajo rendimiento en el trabajo e influye en las personas: en sus actitudes, en su salud física y mental, y en su comportamiento ${ }^{27-29}$. En estas condiciones de desgaste, cambian los factores en los sujetos ${ }^{30}$ : la energía en el trabajo se convierte en desgaste, la implicación con el trabajo en pesimismo, la rentabilidad en poca operatividad.

Algunas cosas aumentan el desgaste de los sujetos en el entorno de trabajo, como tener un trabajo duro ${ }^{31}$ o tener una personalidad que no se adapta fácilmente al medio militar. En este sentido se ha estudiado el papel que juegan algunas variables, como la personalidad tipo $\mathrm{A}^{32,33}$ la dureza psicológica ${ }^{34}$ y la afectividad positiva o la extraversión ${ }^{35,36}$. En un meta-análisis ${ }^{37}$ se estudiaron factores de la personalidad sobre el agotamiento del trabajo y el locus de control como variable predictiva. En otro meta-análisis ${ }^{38}$, consideraron las variables de afectividad positivas y negativas como factores predictores del desgaste en el trabajo.

La necesidad de tomar medidas tanto de evaluación como de prevención de estos factores, que puedan servir para la de toma de decisiones por el mando o los responsables de la organización y como referencia en estudios posteriores, está en 
el origen del planteamiento de este artículo. En este sentido, es importante contar con herramientas y procedimientos de análisis, que permitan la evaluación de los factores de personalidad en distintos contextos laborales y en concreto en el contexto de las FAS y del trabajo en las emergencias y las catástrofes, con el objetivo de facilitar la adaptación del personal, de prevenir factores de riesgo psicológico y en última instancia de hacer a las unidades más operativas.

\section{OBJETIVOS}

Los objetivos que se han planteado en el estudio han sido varios, en una misma línea argumental:

- En primer lugar dar a conocer el trabajo técnico que se realiza desde los servicios de psicología en las unidades de las FAS, a través del estudio de una prueba de evaluación psicológica con personal militar que trabaja en emergencias y catástrofes.

- En segundo lugar mostrar que el trabajo del psicólogo, no sólo es la aplicación de pruebas psicológicas, sino que después hay un trabajo importante, de interpretación y análisis de los resultados obtenidos, que requiere una continua actualización de conocimientos técnicos para poder hacer observaciones más cercanas a la realidad de las personas que prestan su servicio a la sociedad en las unidades de las FAS.

- En último lugar se pretende mejorar los instrumentos de evaluación psicológica utilizados en el seno de las FAS.

\section{MÉTODO}

Como se ha expuesto uno de los propósitos de este artículo es estudiar una prueba que evalúa factores de la personalidad, que se utiliza con el personal que trabaja en situaciones de emergencias y catástrofes en la Unidad Militar de Emergencias (UME) de las FAS españolas.

Se ha realizado el estudio a través del análisis factorial exploratorio que es una técnica de reducción de la dimensionalidad de los datos.

El propósito último del análisis factorial consiste en buscar el número mínimo de dimensiones capaces de explicar el máximo de información contenida en los datos. El análisis factorial consta de cuatro fases características: El cálculo de una matriz capaz de expresar la variabilidad conjunta de todas las variables, la extracción del número óptimo de factores, la rotación de la solución para facilitar su interpretación si es necesario, y la estimación de las nuevas puntuaciones.

La recogida de los datos se llevó a cabo durante el año 2012, se elaboró una base de datos con el programa SPSS y su posterior análisis estadístico.

\section{Participantes}

Se ha utilizado una muestra de militares españoles que ejercen su trabajo en la Unidad Militar de Emergencias (UME), a los que se les aplicó el cuestionario y se les solicitó la autorización para realizar este estudio. La muestra consta de un total de 1.661 militares, (86\%) entre 20 y 40 años, que mayoritariamente convive en pareja $(53,3 \%)$, sin hijos $(65,2 \%)$, con un porcentaje de hombres del 93.2\% la mayoría procedentes de la categoría de tropa no permanente $(66 \%)$ y un porcentaje de cuadros de mando oficiales y suboficiales del $24,2 \%$, con un nivel académico de estudios medios, sólo el $7,4 \%$ eran graduados.

\section{Prueba utilizada}

El Cuestionario de personalidad "VERSIÓN 6RA" que se utiliza en la UME proviene del que fue aprobado para dar cumplimiento al R. D. 944/2001, de 3 de agosto, por el que se aprueba el Reglamento para la determinación de la aptitud psicofísica del personal de las FAS. La prueba es un cuestionario de personalidad que consta de tres escalas de tendencia de respuestas (Aquiescencia, Negaciones y Distorsión), y cinco escalas clínicas (Depresión, Neuroticismo, Psicoticismo, Psicopatía y una escala de Extraversión). El cuestionario inicial elaborado en 1999 fue la primera prueba originada a partir de un banco de ítems cuyos elementos fueron todos previamente aplicados y "filtrados" en muestras de militares españoles. A partir de esta y sus posteriores versiones y estudios se creó la actual versión que se utiliza en esta Unidad.

\section{Operacionalización de los factores}

La prueba de personalidad es un cuestionario de 150 ítems que corresponden a las escalas descritas de 25 ítems cada una. La forma de contestar es en una escala de verdadero y falso en la que se presentan los ítems y el sujeto opta a la respuesta verdadera si el ítem considera que se ajusta a su forma de actual normalmente o falsa si no es así.

Tabla 1. Escalas que componen el $6 R A$.

\begin{tabular}{|lclc|}
\hline \multicolumn{1}{|c}{ Escalas clínicas } & Rango & Escalas de tendencia & Rango \\
\hline Depresión (DE) & $0-25$ & Distorsión (DD) & $0-25$ \\
Neuroticismo (NE) & $0-25$ & Aquiescencia & $0-116$ \\
Psicopatía (PSPT) & $0-25$ & Negaciones & $0-34$ \\
Piscoticismo (PSCT) & $0-25$ & & \\
Extraversión, Sociabilidad (SO) & $0-25$ & & \\
\hline
\end{tabular}

Del total de 150 ítems, 116 se valoran si contesta positivamente al ítem y 34 si la respuesta es negativa. Estas valoraciones hacen que las escalas tengan unos índices que van desde 0 , hasta un máximo de 116 en la escala de aquiescencia, tal como se muestra en la tabla 1. 


\section{Descripción de los factores}

- DEPRESIÓN (DE): se refiere al síndrome caracterizado por la tristeza vital y profunda que envuelve al sujeto hasta afectar todas las esferas de su relación intra e interpersonal. Agrupa unos procesos caracterizados por tristeza, inhibición, culpa, minusvalía y pérdida del impulso vital. - NEUROTICISMO (NE): se refiere al sentimiento de inestabilidad emocional. Es expresión de una intensa conflictividad interna. El sujeto tiene un mal control de sus afectos, por lo que está sometido a una lucha que le ocasionará constantes y penosas tensiones internas, manifestadas en inseguridades, sentimientos de inferioridad y vivencias de culpa y autopunición. El origen biológico, está puesto en la parte simpática del sistema nervioso autónomo, que se ocupa de la lucha y de la huida, y está fuertemente activada por estímulos externos ${ }^{39-41}$.

- PSICOPATÍA (PSPT): el patrón general de este factor es un desprecio hacia los demás, violación de los derechos de los otros, engaño y manipulación. Los sujetos frecuentemente carecen de empatía y tienden a ser insensibles, cínicos y a menospreciar los sentimientos, derechos y penalidades de los demás, se caracteriza por una falta de adaptación a las normas militares y como consecuencia a continuos arrestos y represiones.

- PSICOTICISMO (PSCT): son conductas que indican falta de contacto con la realidad, escasas respuestas emocionales, discordancias afectivas en las relaciones sociales con un progresivo déficit de la voluntad e impulsos y trastornos conductuales. Son sujetos que sin llegar a extremos que llevarían a tomar decisiones de separación del servicio, si que llama la atención su comportamiento en el grupo de referencia. Eysenck sugiere que la dimensión PSCT, está basada en un nivel de activación cortical en el SNC. La impulsividad será un rasgo crucial en la unión entre la condicionabilidad y la personalidad. La impulsividad pertenece a PSCT, y la condicionabilidad está unida a la activación cortical ${ }^{39,42}$.

- SOCIABILIDAD (SOC): equivale al factor Extraversión. Las puntuaciones altas son obtenidas por sujetos que tienen tendencia a ser expansivos, impulsivos y no inhibidos. Son personas que no tienen problemas para relacionarse con desconocidos, son despreocupados, poco exigentes y optimistas. Prefieren el movimiento y la acción a la reflexión. No poseen un gran control sobre sus sentimientos, pierden fácilmente la sangre fría y pueden ser agresivos. No son personas en las que siempre se pueda confiar. Se caracterizan por un bajo nivel de activación cortical en comparación con la personalidad introvertida. Para conseguir un óptimo nivel de activación, necesitan más excitación y estímulos en su medio ambiente ${ }^{40-43}$.

- DISTORSIÓN (DIST): es un factor de control que indica el grado de sinceridad con que responde el sujeto o el nivel de distorsión de las respuestas con el objeto de dar una mejor imagen social.

- AQUIESCENCIA (AQ). Estima el grado de aquiescencia, es decir, el número excesivo de respuestas SI.

- NEGACIONES (NG). Estima el grado de negaciones, es decir, el número excesivo de respuestas NO.

\section{RESULTADOS}

\section{Análisis de la prueba utilizada}

En el primer estudio exploratorio se han observado índices adecuados para poder realizar el análisis factorial propuesto: en la prueba de esfericidad de Bartlett, se rechaza la hipótesis de esfericidad. El índice de Crombach en las escalas clínicas es de 0,076. El índice de adecuación de la muestra de Kaiser-MeyerOlkin se encontraba por encima del 0,50 recomendado (KMO $=0,711)$.

\section{Extracción de los factores}

En la extracción de los factores clínicos se observó que tanto en la solución sin rotar como la solución rotada, se mantenían únicamente dos factores en la prueba, con un Alfa de Cronbach de 0,7 . En el primer factor se incluían las variables de depresión, neuroticismo y psicoticismo y se explicaba el $47 \%$ de la varianza y en el segundo factor las variables de psicopatía y sociabilidad, explicando el 23,4\% de la varianza. Se decidió por tanto llamar al primer factor "emocional" y al segundo factor "social". Los eigenvalue siguen la regla de regla Kaiser (eigen $>1$ ) y son de 2,37 y 1,17 correspondientes al primer y segundo factor respectivamente.

\section{Solución sin rotar}

Se puede observar a través de la matriz y el gráfico de componentes, confirmando lo dicho anteriormente, que lo más adecuado es adoptar dos factores principales en los que las saturaciones de las variables se expresan en las siguientes matrices de componentes. Para asignar los ítems a los factores se consideraron las cargas factoriales superiores a 0,40 (Tabla 2 y Figura 1).

Tabla 2. Matriz sin rotar.

\begin{tabular}{|lcc|}
\hline \multicolumn{1}{|c}{ M Componentes } & $\mathbf{1}$ & $\mathbf{2}$ \\
\hline DE &, 804 &,- 131 \\
NEURO &, 847 &,- 092 \\
PCISMO &, 823 &, 200 \\
PTIA &, 405 &, 757 \\
SO &,- 393 &, 732 \\
\hline
\end{tabular}

\section{Solución rotada}

En la siguiente matriz y gráfico se puede observar la solución rotada mediante el procedimiento de rotación ortogonal Varimax con Kaiser. Se ha elegido este método por que se consigue respetar la independencia entre los factores estudiados en la solución inicial y minimiza el número de variables que tienen saturaciones altas en cada factor. Simplifica la interpretación de los factores optimizando la solución por columna (Tabla 3 y Figura 2). Se observa que los ítems se distribuyen según sus cargas de manera similar a como se han observado en la matriz sin rotar (Tabla 4). 


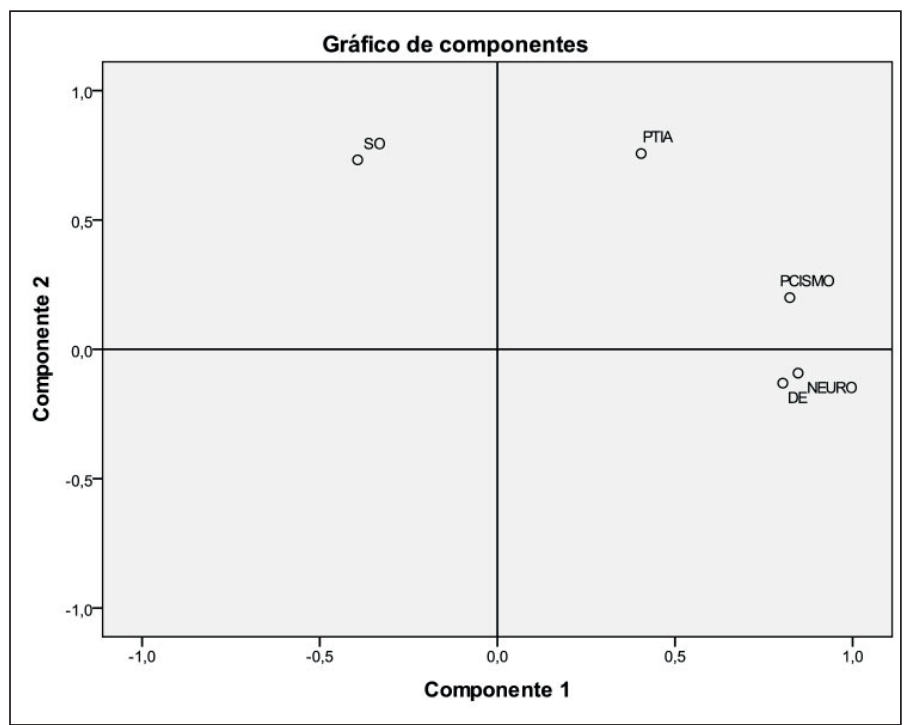

Figura 1. Matriz sin rotar.

Tabla 3. Rotación Varimax.

\begin{tabular}{|lcc|}
\hline \multicolumn{1}{r}{$\begin{array}{c}\text { Rot. varimax } \\
\text { con Kaiser }\end{array}$} & \multicolumn{2}{c|}{ Componente } \\
\hline DE &, 808 & $\mathbf{2}$ \\
NEURO &, 850 &,- 097 \\
PCISMO &, 814 &,- 057 \\
PTIA &, 373 &, 773 \\
SO &,- 423 &, 715 \\
\hline
\end{tabular}

Tabla 4. Matriz de transformación.

\begin{tabular}{|ccc|}
\hline \multicolumn{3}{|c|}{ Matriz de transformación } \\
\hline Componente & $\mathbf{1}$ & $\mathbf{2}$ \\
$\mathbf{1}$ &, 999 &, 042 \\
$\mathbf{2}$ &,- 042 &, 999 \\
\hline
\end{tabular}

En este caso se puede deducir que se mantiene los ángulos entre las variables a pesar de rotar la solución. Se determinan por tanto dos factores clínicos: en el primer factor se incluyen las escalas de Depresión, Neuroticismo y Psicoticismo. Y en el segundo factor se incluyen las escalas de Psicopatía y Sociabilidad. Se entiende que el primer factor está más relacionado con el estado de ánimo, la inestabilidad emocional y con el sentido de la realidad de los sujetos y se le denomina: "EMOCIONAL". El segundo factor está más relacionado con lo social: por un lado la extraversión y por otro la psicopatía, este último concepto muy relacionado con lo antisocial ${ }^{44,45}$ pero que han mostrado unos índices de relación positivos $\mathrm{y}$ significativos entre ambos $(0,134)$ por lo que al segundo factor se le denomina: "SOCIAL". Consiguiendo dar sentido al principio básico de funcionamiento de las personas, al hacer un tratamiento de las mismas como una totalidad o sistema bio-psico-social ${ }^{46}$.

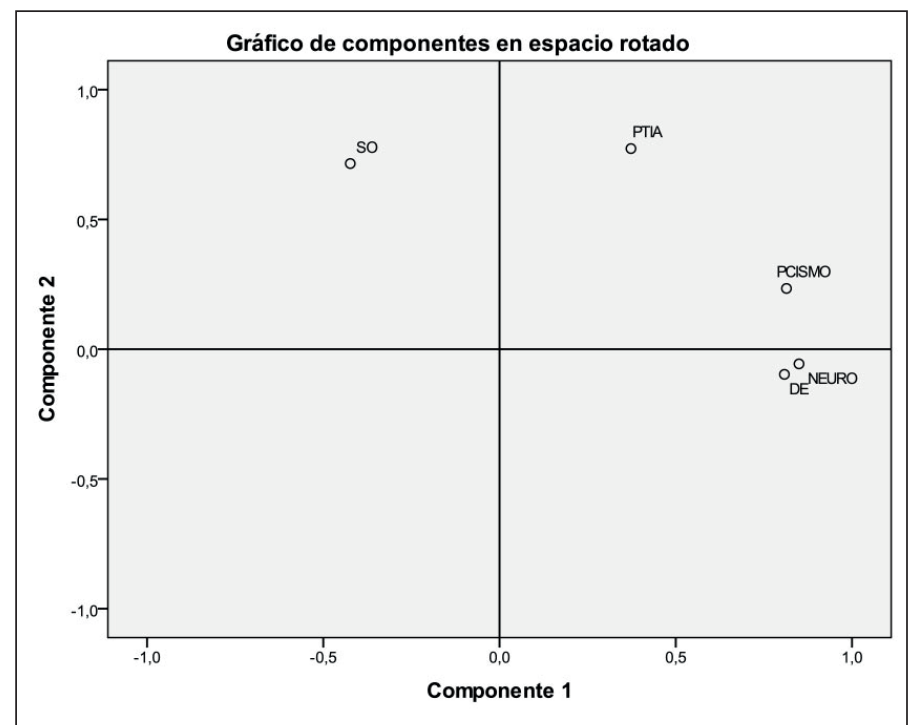

Figura 2. Rotación Varimax.

\section{Estimación de las puntuaciones}

Una vez alcanzada la solución factorial final, suele resultar interesante baremar y obtener una estimación de las puntuaciones de los sujetos en cada uno de los factores resultantes de la extracción, a fin de valorar la situación relativa de cada sujeto en esas nuevas "dimensiones ocultas" capaces de resumir la información contenida en las variables originales. Para ello se ha utilizado el método de Regresión. Método de estimación de las puntuaciones factoriales en el que las estimaciones resultantes tienen una media de cero y una varianza igual al cuadrado de la correlación múltiple entre las puntuaciones factoriales estimadas y los valores factoriales verdaderos. Las puntuaciones factoriales estimadas con este método pueden estar correlacionadas incluso cuando los factores son ortogonales (Tabla 5).

\section{DISCUSIÓN}

Tal como señala la propia Unión Europea los factores psicológicos relacionados con el trabajo son convenientes y necesarios identificarlos, evaluarlos y controlarlos con el fin de evitar sus riesgos asociados para la salud y la seguridad en el trabajo ${ }^{47}$.

Estos factores no siempre son perjudiciales sino que tienen un doble efecto positivo y negativo ${ }^{48}$. Las formas acertadas de cultura en la organización, de clima laboral o de liderazgo y de mando en las unidades militares, afectan a la salud positivamente generando desarrollo individual, bienestar para las personas y beneficios para la organización. Cuando estos factores son disfuncionales, provocan tensiones, malestar, problemas de adaptación, respuestas de estrés negativas y pasan a denominarse factores de riesgo ${ }^{49}$.

Un problema con este tipo de variables psicológicas relacionadas con la personalidad, es la dificultad para encontrar unidades de medida adecuadas ${ }^{50}$. A través de trabajos como el que se presenta en este artículo se pueden encontrar indicadores más correctos y operacionalizables ${ }^{51}$, que se puedan tener en cuenta en las Unidades Militares. 
Tabla 5. Descriptivos de los nuevos factores de personalidad.

\begin{tabular}{|c|c|c|c|}
\hline \multicolumn{4}{|c|}{ Estadísticos de los nuevos factores de personalidad } \\
\hline \multirow[b]{2}{*}{ Media } & & $\begin{array}{c}\text { Factor I } \\
\text { Emocional }\end{array}$ & $\begin{array}{c}\text { Factor II } \\
\text { Social } \\
\end{array}$ \\
\hline & & 0 & 0 \\
\hline \multicolumn{2}{|l|}{ Mediana } & $-0,31$ & $-0,06$ \\
\hline \multicolumn{2}{|l|}{ Moda } & $-0,90$ & $-0,36$ \\
\hline \multicolumn{2}{|l|}{ Desv. típ. } & 1 & 1 \\
\hline \multicolumn{2}{|c|}{ Asimetría } & 2,17 & 0,47 \\
\hline \multicolumn{2}{|c|}{ Error típ. de asimetría } & 0,06 & 0,06 \\
\hline \multicolumn{2}{|c|}{ Curtosis } & 6,14 & 0,48 \\
\hline \multicolumn{2}{|c|}{ Error típ. de curtosis } & 0,12 & 0,12 \\
\hline \multirow{9}{*}{ Percentiles: } & 10 & $-0,86$ & $-1,27$ \\
\hline & 20 & $-0,72$ & $-0,80$ \\
\hline & 30 & $-0,59$ & $-0,50$ \\
\hline & 40 & $-0,46$ & $-0,26$ \\
\hline & 50 & $-0,31$ & $-0,06$ \\
\hline & 60 & $-0,11$ & 0,16 \\
\hline & 70 & 0,18 & 0,38 \\
\hline & 80 & 0,52 & 0,72 \\
\hline & 90 & 1,21 & 1,30 \\
\hline
\end{tabular}

Previo al análisis factorial se han observado los índices estadísticos que permiten realizar el análisis propuesto. Las soluciones factoriales presentadas, mantenían dos factores de personalidad, en el primer factor se incluían las variables de depresión, neuroticismo y psicoticismo de la prueba y en el segundo factor las variables de psicopatía y sociabilidad, denominando a estos factores como: "emocional" y "social", en ambos casos los índices de varianza explicada no son muy altos, sobre todo en el caso del factor social, y el índice de falibilidad de la prueba es mejorable. Corrigiendo estos factores se podría evaluar de forma más precisa y por tanto realizar mejores predicciones sobre las conductas que están asociadas biológica, psicológica o socialmente a los factores de personalidad.

Está demostrado que hay relación entre los rasgos de la personalidad y otros factores de ámbito personal, social y de las organizaciones. Estos rasgos influyen en la calidad de las relaciones en el entorno de trabajo interpesonal ${ }^{52,53}$, en actitudes de compromiso en el trabajo y en la comunidad ${ }^{54-59}$, además de condicionar la vida personal y la salud en aspectos físicos y psicológicos ${ }^{60,61}$.

En estudios realizados en la UME, se ha observado que la variable personal "emocional" se relaciona con variables como el desgaste en el trabajo y con estresores laborales como el estrés de rol. La variable de personalidad "social" sin embargo no se relacionó significativamente con las variables de estrés de rol y muy débilmente con las variables de desgaste analizadas, incluso la relación es negativa y no significativa en cuanto a la variable "realización profesional”. Se puede sugerir aquí según los resultados comentados, que los sujetos que obtienen puntuaciones altas en el componente personal "emocional" se podrían considerar más vulnerables ${ }^{24,25}$.

El estudio que se ha presentado aporta una visión sobre la forma de trabajo de los psicólogos en las Unidades Militares, que va un poco más allá de la pura aplicación de tests psicológicos. Se ha tenido en cuenta que en los últimos tiempos ${ }^{62}$ se ha desarrollado un cambio importante a la hora de abordar los problemas en el entorno de trabajo, incluyendo otras variables como las posibilidades de desarrollo personal y laboral, lo que ha supuesto un cambio conceptual importante ${ }^{63}$. Sin embargo el estudio de los recursos de tipo personal ha sido menos sistemático y minucioso ${ }^{64}$.

Los aspectos personales juegan un importante papel en los procesos de bienestar laboral. En un modelo reciente que nos puede servir de referencia, se subraya la importancia de los recursos de las personas en lo que se denomina Capital Psicológico y propone cuatro variables personales positivas: La autoeficacia, el optimismo, la esperanza y la resiliencia ${ }^{65}$, que aumentan la capacidad para manejar situaciones difíciles y junto a la proactividad personal favorece el bienestar y el desempeño laboral superior. Unido a estos constructos se puede incluir otro ya clásico, el de personalidad resistente o capacidad de percibir las acciones como parte del desarrollo personal a través de tres factores: compromiso, control y reto ${ }^{66}$.

El medio militar implica trabajos en situaciones difíciles, con un alto contenido de estrés y por tanto requiere profesionales con una estabilidad y unas cualidades personales que sean aptos para llevar a cabo las misiones en las mejores condiciones psicofísicas posibles. Esto requiere una evaluación y unos instrumentos de precisos, para conseguir un máximo acercamiento a la realidad. De esta forma se consigue mejorar el ajuste y la adaptación de la persona al puesto de trabajo, evitando parte de los problemas psicológicos de adaptación al medio militar y en última instancia mejorando la operatividad de las unidades militares en el cumplimiento de sus misiones.

Por tanto se propone con este artículo seguir investigando y actualizando los instrumentos que se utilizan en una actitud de mejorar continuamente. En el sentido de que sean más precisos, que la información que aporten sea más cercana a la realidad, y que aporten una información útil para el desarrollo de los objetivos operativos de unas FAS del futuro.

\section{BIBLIOGRAFÍA}

1. Pelechano, V . (1993). Personalidad: Un enfoque histórico - conceptual. Valencia. Promolibro.

2. Millon, T. y Davis, R. (1998). Trastornos de la personalidad. Más allá del DSM-IV. Barcelona: Masson.

3. DSM IV TR (2002). Manual de Diagnóstico y Estadístico de los trastornos mentales. Barcelona: Masson.

4. Eysenck, H.J. (1947). Dimensions of personality. London: Routledge and Kegan Paul.

5. Eysenck, H.J. y Eysenck, M.W. (1985). Personality and individual differences: A natural science approach. New York: Plenum.

6. Eysenck, H.J. (1990b). Genetic and environmental contributions to individual differences: The three major dimensions of personality. Journal of Personality, 58: 245-261.

7. Eysenck, H. J. y Eysenck, M. (1985). Personality and Individual Differences, N.Y. Plenum Press.

8. Eysenck, H. J. (1967). The biological basis of personality. Springfield, III, Charles C. Thomas. (Versión en español de 1982. Fundamentos biológicos de la personalidad. Barcelona: Fontanella).

9. Ballenger, J. C., et al. (1983). Biochemical correlates of personality traits in normals: An exploratory study. Personality and Individual Differences, 4: 615-625.

10. Labrador, F.J. (1992). El estrés: Nuevas técnicas para su control. Madrid: Temas de Hoy.

11. Labrador, F. J., y Crespo, M. (1993). Estrés. Trastornos psicofisiológicos. Madrid: Eudema. 
12. Corr, P.J. (2001). Testing problems in J.A. Gray's personality theory: a commentary on Matthews and Gilliland (1999). Personality and Individual Differences, 30: 333-352.

13. Matthews, G. y Gilliland, K. (1999). The personality theories of H.J. Eysenck and J.A. Gray: a comparative review. Personality and Individual differences, 26: 583-626.

14. Rutter, M., Giller, H. y Hagell, A. (2000). La conducta antisocial de los jóvenes. Cambridge University Press.

15. Herrero, O., y Colom, R. (2006). ¿Es verosímil la teoría de la delincuencia de David Lykken?. Psicothema, 18(3): 374-377.

16. Roberston, I.T., Cooper, CL. Y Williams, J. (1990). The Validity of the Occupational Stress Indicator. Work and Stress, 4: 29-39.

17. Eysenck, H.J. (1977). Crime and personality. London: Routledge y Kegan Paul.

18. Eysenck, H.J. (1992). The definition and measurement of psychoticism. Personality and Individual Differences, 13: 757-785.

19. Eysenck, H.J. y Gudjonsson, G. (1989). The causes and cures of criminality. New York: Plenum Press.

20. Aluja, A. (1991). Personalidad desinhibida, agresividad y conducta anti-social. Barcelona: PPU.

21. Rebollo, I., Herrero, O. y Colom, R. (2002). Personality differences between imprisoned and non imprisoned people: evidence from the EPQ-R. Psicothema, 14(3): 540-543.

22. Zuckerman, M. (1979). Sensation seeking: beyond the optimal level of arousal. Hilldale: Erlbaum.

23. Hare, R.D. (1999). La naturaleza de los psicópatas: algunas observaciones para entender la violencia depredadora humana. Ponencia presentada a la Cuarta Reunión Internacional sobre Sociología y Biología de la Violencia. Centro Reina Sofía para el Estudio de la Violencia.

24. Lykken, D.T. (1995): The antisocial personalities. New Jersey: LEA

25. Gray, J.A. (1987). The psychology of fear and stress. Cambridge: University of Cambridge Press.

26. Instituto Nacional de Seguridad e Higiene en el Trabajo Manual de procedimientos de Prevención de Riesgos laborales http://www.insht.es (revisada Enero 2013).

27. Cordes, C. L. y Dougherty, T. W. (1993). A review and an integration of research on job burnout. Academy of Management Review, 18: 621-656.

28. Maslach, C., y Schaufeli, W. B. Leiter, M. P. (2001). Job burnout. In S. T. Fiske, D. L. Schacter and C. Zahn-Waxler (Eds.), Annual Review of Psychology, 52: 397-422.

29. Maslach, C. y Goldberg, J. (1998). Prevention of burnout: New perspectives. Applied and Preventive Psychology, 7, 63_74.

30. Maslach, C. and Leiter, M. P. (1997). The Truth About Burnout: How Organizations Cause Personal Stress and What to Do About It, Jossey-Bass.

31. Alarcon, G., Eschleman, K. J. y Bowling, N. A. (2009). Relationship between Personality Variables and Burnout: A Meta-Analysis. Work and Stress, Vol. 23, No. 3, July-September. 244-263.

32. Ganster, D. C. (1986). Type A behavior and occupational stress. Journal of Organizational Behavior Management 8: 61-84.

33. Kirmeyer, S. L. (1988). Coping with competing demands: interruption and the Type A pattern. Journal of Applied Psychology 73: 621-629.

34. Rush, M. C., Schoel, W. A. and Barnard, S. M. (1995). Psychological resiliency in the public sector: "hardness" and pressure for change. Journal of Vocational Behavior 46: 17-39.

35. Iverson, R. D., Olekalns, M. and Erwin, P. J. (1998). Affectivity, organizational stressors, and absenteeism: a causal model of burnout and its consequences. Journal of Vocational Behavior 52: 1-23.

36. Kahn, J. H., Schneider, K. T., Jenkins-Henkelman, T. M., Moyle, L. L. (2006). Emotional social support and job burnout among high-school teachers: is it all due to dispositional affectivity? Journal of Organizational Behavior 27: 793-807.175.

37. Sorensen, K. L., Eby, L. T. (2006). Locus of control at work: A meta-analysis. Journal of Organizational Behavior, 27: 1057-1087.

38. Thoresen, C. J., Kaplan, S. A., Barsky, A., Warren, C. R., and de Chermont, K. (2003). The affective underpinnings of job perceptions and attitudes: A metaanalytic review and integration. Psychological Bulletin, 129: 914_945.

39. Gudjonsson, G.H. (1997). Accusations by adults of childhood sexual abuse: A survey of the members of the British false memory society (BFMS). Applied Cognitive Psychology, 11: 3-18.

40. Eysenck, H.J. (1977). Crime and personality. Londres: Paladin.
41. Eysenck, H.J. y Gudjonson, G. (1989b). The causes and cures of criminality. New York: Plenum Press.

42. Eysenck, H.J y Eysenck, B.G. (1998). EPQ: Cuestionario de personalidad para niños (EPQ-J) y adultos (EPQ-A). Vol, 97 de Publicaciones de psicología aplicada.

43. 43 Blackburn, R. (1993). The psychology of criminal conduct. Nueva York: Wiley.

44. Hare, R.D. (1991). The Hare Psychopathy Ckecklist-Revised. Toronto: MultiHealth Systems.

45. Hare, R.D. (2001). Psychopaths and their nature. En A. Raine y J. Sanmartín (Eds.): Violence and psychopathy. New York: Kluwer Academic/Plenum Publishers. 5-34.

46. Engel, G. (1977). The need for a new medical model: A challenge for biomedicine. Science, 196: 129-136.

47. European Agency for Safety and Health at Work. Annual Report (2007). UEOSHA. European Social Partners.

48. Kalimo, R., El-Batawi, M., and Cooper, C. L. (1988). Los factores psicosociales en el trabajo y su relación con la salud. Ginebra: OMS.

49. Benavides, F., Gimeno, D., Benach.J., Martinez, J. M., Jarque, S., Berra, A (2002). Descripción de los factores de riesgo psicosocial en cuatro empresas. Gaceta Sanitaria, 16: 222-229.

50. OIT (1986). Factores psicosociales en el trabajo: reconocimiento y control. Ginebra: OIT.

51. Roozeboom, M. B., Houtman, I.,Van den Bossche, S. (2008). Monitoring Psychosocial Risks at work. I. En S.Leka, T. Cox (Eds.),The European Framework for psychosocial Risk management: PRIMA-EF 17-36. Nottingham: I-WHO.

52. Eisenberg, N., Fabes, R.A., Guthrie, I.K. Reiser, M. (2000). Dispositional emotionality and regulation: Their role in predicting quality of social functioning. Journal of Personality and Social Psychology, 78: 136-157.

53. Karney, B.R. y Bradbury, T.N. (1995). The longitudinal course of marital quality and stability: A review of theory, method, and research. Psychological Bulletin, 118: 3-34.

54. Hopwood, C.J., Morey, L.C., Ansell, E.B, Grilo, C.M., Sanislow, C.A., Skodol, A.E. (2009). The convergent and discriminant validity of Five-Factor traits: Current and prospective social, work, and recreational dysfunction. Journal of Personality Disorders, 23(5): 466-476.

55. Judge, T.A., Higgins, C.A., Thoresen, C.J. y Barrick, M.R. (1999). The Big Five personality traits, general mental ability, and career success across the life span. Personnel Psychology, 52(3): 621-652.

56. Barrick, M.R. y Mount, M.K. (1991). The Big Five personality dimensions and job performance: A meta-analysis. Personnel Psychology, 44(1): 1-26.

57. Caspi, A., Roberts, B.W. y Shiner, R.L. (2005). Personality development: Stability and change. Annual Review of Psychology, 56: 453-484.

58. Judge, T.A. y Kammeyer-Mueller, J.D. (2007). Personality and career success. En H.P. Gunz y M.A. Peiperl (Eds.), Handbook of career studies 59-78. Thousand Oaks, CA.

59. Ozer, D.J. y Benet-Martínez, V. (2006). Personality and the prediction of consequential outcomes. Annual Review of Psychology, 57: 401-421.

60. Roberts, B.W., Kuncel, N.R., Shiner, R., Caspi, A. y Goldberg, L.R. (2007). The power of personality: The comparative validity of personality traits, socioeconomic status, and cognitive ability for predicting important life outcomes. Perspectives on Psychological Science, 2(4): 313-345.

61. Widiger, T.A. y Trull, T.J. (2007). Plate tectonics in the classification of personality disorder: Shifting to a dimensional model. American Psychologist, 62: 71-83.

62. Moreno-Jiménez, B., Garrosa, E., Corso, S., Boada; M., y Rodríguez-Carbajal, R,. (2012). Personalidad resistente y capital psicológico: las variables personales positivas y los procesos de agotamiento y vigor. Psicothema 2012. 24(1): 79-86.

63. Nelson, D.L., y Cooper, C.L. (2007). Positive Organizacional Psychology. London: Sage Publications. Randles,

64. Swider, B.W., y Zimmerman, R.D. (2010). Born to burnout: A meta-analytic path model of personality job burnout and work outcomes. Journal of Vocational Behaviour, 76: 487-506.

65. Luthans, F., Youseff, C.M., y Avolio, B.J. (2007). Psychological capital: Developing the human competitive edge. Oxford: Oxford University Press.

66. Maddi, S.R., Kobasa, S.C., y Kahn, S. (1982). Hardiness and health: A prospective study. Journal of Personality and Social Psychology, 42: 168-177. 